\title{
Inhibition of VEGF mediated corneal neovascularization by anti-angiogenic peptide nanofibers
}

\author{
Berna Senturk a , M. Ozgur Cubuk b, M. Cuneyt Ozmen b , Bahri Aydin b, **, \\ Mustafa O. Guler a , Ayse B. Tekinay ${ }^{\mathrm{a}, \text { * }}$ \\ a Institute of Materials Science and Nanotechnology, National Nanotechnology Research Center (UNAM), Bilkent University, Ankara, 06800, Turkey \\ b Department of Ophthalmology, School of Medicine, Gazi University, Ankara, 06100, Turkey
}

\section{A R T I C L E I N F O}

\section{Article history:}

Received 14 June 2016

Accepted 29 August 2016

Available online 30 August 2016

\section{Keywords:}

Corneal neovascularization

Anti-angiogenesis

Peptide amphiphiles

Neuropilin-1

\begin{abstract}
A B S T R A C T
Atypical angiogenesis is one of the major symptoms of severe eye diseases, including corneal neovascularization, and the complex nature of abnormal vascularization requires targeted methods with high biocompatibility. The targeting of VEGF is the most common approach for preventing angiogenesis, and the LPPR peptide sequence is known to strongly inhibit VEGF activity by binding to the VEGF receptor neuropilin-1. Here, the LPPR epitope is presented on a peptide amphiphile nanofiber system to benefit from multivalency and increase the anti-angiogenic function of the epitope. Peptide amphiphile nanofibers are especially useful for ocular delivery applications due to their ability to remain on the site of interest for extended periods of time, facilitating the long-term presentation of bioactive sequences. Consequently, the LPPR sequence was integrated into a self-assembled peptide amphiphile network to increase its efficiency in the prevention of neovascularization. Anti-angiogenic effects of the peptide nanofibers were investigated by using both in vitro and in vivo models. LPPR-PA nanofibers inhibited endothelial cell proliferation, tube formation, and migration to a greater extent than the soluble LPPR peptide in vitro. In addition, the LPPR-PA nanofiber system led to the prevention of vascular maturation and the regression of angiogenesis in a suture-induced corneal angiogenesis model. These results show that the anti-angiogenic activity exhibited by LPPR peptide nanofibers may be utilized as a promising approach for the treatment of corneal angiogenesis.
\end{abstract}

(c) 2016 Elsevier Ltd. All rights reserved.

\section{Introduction}

Angiogenesis is the formation of new blood vessels from preexisting networks and is tightly regulated through the activation of endothelial cells, local degradation of the extracellular matrix and vascular basement membranes, and stabilization of newly formed vessels through the proliferation and migration of the native cell population [1]. Endothelial cell activation is critical for the modulation of this process and is determined by the positive (angiogenic) and negative (anti-angiogenic) regulators of angiogenesis, including VEGF, aFGF and bFGF [2]. Abnormalities in the production of these growth factors and their downstream elements may result in excessive or insufficient blood vessel formation,

\footnotetext{
* Corresponding author.

** Corresponding author.

E-mail addresses: baydunus@yahoo.com (B. Aydin), moguler@unam.bilkent.edu. $\operatorname{tr}$ (M.O. Guler), atekinay@bilkent.edu.tr (A.B. Tekinay).
}

potentially interfering with the function of affected tissues.

Pathological angiogenesis occurs in many disorders, including corneal neovascularization, which causes visual impairment through the loss of ocular transparency [3]. Vascular endothelial growth factor (VEGF) and its receptors have been found to be significant modulators of angiogenesis, and their inhibition is an effective means of corneal neovascularization treatment. Bevacizumab, a clinically approved drug, is a recombinant humanized monoclonal IgG1 antibody that binds to and inhibits the biological activity of human VEGF both in vitro and in vivo [4]. However, while bevacizumab therapy has been shown to facilitate the regression of corneal neovascularization in animal studies and clinical reports, its effectiveness is limited by safety issues and the recurrence of neovascularization in certain cases [5]. High costs, difficulties of manufacturing and short half-lives are further disadvantages of bevacizumab and other antibody drugs [6]. Therefore, new therapeutics and formulations capable of eliminating these deficiencies are urgently needed. 
Peptide-mediated targeting strategies exhibit considerable potential for inhibiting the activity of angiogenic factors. Selfassembling peptide amphiphile molecules consist of a hydrophilic head group containing bioactive epitopes and $\beta$-sheet forming sequences, which is chemically conjugated to a hydrophobic tail region that further assists in the assembly process [7]. They have a wide range of applications, such as tissue engineering, regenerative medicine, diagnostic tools, drug delivery and functional biomaterials [8-10]. They are favorable over proteins and other small molecules due to their stability and ease of preparation and purification. In addition, several reports have recently shown that peptide systems can be used as angiogenesis inhibitors [11-13].

The LPPR peptide sequence has previously been identified by the phage display technique and was shown to bind specifically to the VEGF receptor, neuropilin-1 (NRP-1) [14]. This binding strongly inhibits the activation of NRP- 1 by VEGF 165 , and the LPPR sequence is responsible for $75 \%$ of this inhibition [15]. In addition, the peptide was able to significantly decrease tumor growth through the inhibition of angiogenesis in an in vivo breast cancer model, while a similar peptide sequence, $D(L P R)$, exhibited promising results for the treatment of retinopathy of prematurity $[16,17]$. Since the LPPR peptide sequence is essential for the inhibition of VEGF activity, we utilized this peptide sequence on a self-assembled peptide amphiphile nanofiber system to increase epitope presentation and create a highly effective anti-angiogenic agent.

The Lauryl-VAAGKKGLPPR-Am peptide amphiphile molecule was designed and synthesized to form a $\beta$-sheet containing supramolecular structure, thereby increasing its solubility in water and presenting the functional epitope on a nanofiber system. The self-assembly process, secondary structure and cellular localization of the peptide nanofibers were characterized in detail. The capacity of the peptide nanofiber formulation to inhibit blood vessel formation was then evaluated by using in vitro angiogenesis, scratch healing and tube formation assays. In addition, the effect of nanofibers was studied in an in vivo suture induced corneal neovascularization model. The efficiency of the bioactive PA nanofibers was found to be comparable to the commercially available drug bevacizumab and superior to the soluble peptide formulation.

\section{Material and methods}

\subsection{Materials}

All 9-fluorenylmethoxycarbonyl (Fmoc) and tert-butoxycarbonyl (Boc) protected amino acids, [4-[ $\alpha$-(20,40-dimethoxyphenyl) Fmocaminomethyl]phenoxy]acetamidonorleucyl-MBHA resin (Rink amide MBHA resin), and Fmoc-Asp(OtBu)-Wang resin were purchased from NovaBiochem. 2-(1H-Benzotriazol-1-yl)-1,1,3,3tetramethyluronium hexafluorophosphate (HBTU) was purchased from ABCR. Antibodies were purchased from Abcam and Millipore. Matrigel $^{\mathrm{TM}}$ (growth factor reduced) was purchased from BD Biosciences (356230), Live-Dead viability assay kit from Thermo Fisher Scientific (L3224) and ELISA-based BrdU proliferation assay kit from Roche (11647229001). All other materials were obtained from Invitrogen, Fisher, Merck, Alfa Aesar, and Sigma-Aldrich. All materials were analytical grade.

\subsection{Peptide synthesis and nanofiber formation}

Peptide amphiphile (PA) molecules used in this study were synthesized by solid phase peptide synthesis. Rink amide MBHA resin (for Ac-LPPR-Am, LPPR-PA, scrambled-PA and $K_{3}-P A$ ) and Fmoc-Glu(OtBu)-Wang resin (for E-PA) were used as solid support. Coupling reactions of amino acids were performed with 2 equivalents of Fmoc protected amino acid, 1.95 equivalents of HBTU and 3 equivalents of $\mathrm{N}, \mathrm{N}$-diisopropylethylamine (DIEA) in DMF for $3 \mathrm{~h}$. Fmoc removals were performed with $20 \%$ piperidine/dimethylformamide (DMF) solution for $20 \mathrm{~min}$.

All peptides were cleaved from the resin using a mixture of trifluoroacetic acid (TFA), triisoproplysilane (TIS), and water at a ratio of 95: 2.5: 2.5 for $2.5 \mathrm{~h}$. Excess TFA was removed by rotary evaporation and the sample was triturated with ice-cold diethyl ether. Diethyl ether was then removed and the pellet was dissolved in water for freeze-drying. Peptide samples were analyzed and purified by reverse phase preparative HPLC on an Agilent 6530 Accurate-Mass Q-TOF LC/MS equipped with an Agilent 1200 HPLC.

The bioactive LPPR-PA nanofiber was formed by mixing LPPR-PA and E-PA at 1:1 molar ratio, while the non-bioactive $\mathrm{K}_{3}-\mathrm{PA} / \mathrm{E}-\mathrm{PA}$ control nanofiber was similarly formed by mixing $\mathrm{K}_{3}-\mathrm{PA}$ and $\mathrm{E}-\mathrm{PA}$ at 1:1 molar ratio. Scrambled-PA was also mixed with E-PA at 1:1 molar ratio. Net charges were +1 for all nanofibers and LPPR peptide (Fig. S1). Peptide mixtures and solutions were adjusted to $\mathrm{pH}$ 7.4 with $\mathrm{HCl}$ or $\mathrm{NaOH}$ prior to use.

Circular dichroism (CD) measurements were carried out at $100 \mu \mathrm{M}$ peptide concentrations in water and a $1-\mathrm{mm}$ path length quartz cuvette. Peptides were vortexed to ensure mixing and the $\mathrm{pH}$ was adjusted with $\mathrm{HCl}$ or $\mathrm{NaOH}$ to 7.4. Measurements were acquired at room temperature at $500 \mathrm{~nm} / \mathrm{min}$ scanning speed, $1 \mathrm{~nm}$ bandwidth and $0.1 \mathrm{~nm}$ data pitch using a J-815 Jasco spectrophotometer. The 190-300 nm spectral regions were monitored for the analysis of the secondary structures of peptides.

Transmission electron microscopy (TEM) and scanning transmission electron microscopy (STEM) samples were prepared at a final concentration of $100 \mu \mathrm{M}$ of mixtures of positively and negatively charged peptides in water. $5 \mu \mathrm{L}$ of samples were cast onto TEM grids and incubated for $5 \mathrm{~min}$. Samples were then stained with $2 \mathrm{wt} \%$ uranyl acetate solution and washed twice with a drop of MilliQ water. Imaging was performed by using a FEI Tecnai G2 F30 TEM with electron energy of $100 \mathrm{kV}$.

Sample solutions for AFM were dropped on a glass surface and mixed by pipetting. $25 \mu \mathrm{L}$ of $100 \mu \mathrm{M}$ scrambled-PA and equimolar concentrations of E-PA were used for sample preparation. Peptides were dissolved in water and solutions were adjusted to $\mathrm{pH} 7.4$ for nanofiber formation. After $30 \mathrm{~s}$, excess solution was removed using dust-free tissue paper, and the precipitated PA networks were airdried in a convection oven. Contact mode atomic force microscopy (AFM) was performed using a MFP-3D AFM (Asylum Research, US). Images were taken in air using a SiNi tip with a nominal spring constant of $0.2 \mathrm{~N} / \mathrm{m}$, under a scan rate of $0.5 \mathrm{~Hz}$ and at a resolution of $512 \times 512$.

\subsection{In vitro cell culture studies}

Human umbilical vein endothelial cells (HUVECs) were cultured in a humidified, $37{ }^{\circ} \mathrm{C}, 5 \% \mathrm{CO}_{2}$ incubator using $75 \mathrm{~cm}^{2}$ polystyrene cell culture flasks containing low glucose Dulbecco's modified Eagle's medium (DMEM) supplemented with $10 \%$ fetal bovine serum (FBS, Gibco), $1 \%$ penicillin/streptomycin (P/S) and $2 \mathrm{mM} \mathrm{L-}$ glutamine.

Viability of HUVECs on soluble nanofibers was tested using LiveDead viability assay. HUVECs were seeded in 96-well plates at a density of $2 \times 10^{3}$ cells/well. After $24 \mathrm{~h}$ of peptide incubation, cells were stained with Calcein AM and ethidium homodimer reagents at $2 \mu \mathrm{M}$ and $4 \mu \mathrm{M}$ concentrations, respectively. After $30 \mathrm{~min}$ of incubation, cells were observed under fluorescence microscope, and live and dead cells were counted by using Image (NIH) software. Viability was assessed by calculating the ratio of live cells to total cell numbers. Experiments were carried out with $n=4$ and images were taken from 5 different locations per well.

Cell proliferation was evaluated using a colorimetric ELISA- 
based BrdU assay (Cell Proliferation ELISA, BrdU; Roche) according to the manufacturer's instructions. Briefly, HUVECs were seeded onto a 96-well plate at a density of $5 \times 10^{3}$ cells/well. Cells were subsequently incubated with VEGF $(100 \mathrm{ng} / \mathrm{mL})$ in the presence or absence of peptides for $24 \mathrm{~h}$ at $37^{\circ} \mathrm{C}$, and the extent of BrdU incorporation was quantified by spectrophotometry. Each group was tested at least in triplicate, and the assays were repeated a minimum of three times. The $\mathrm{IC}_{50}$ of cell proliferation was calculated by taking untreated cells as maximum and using GraphPad program.

For in vitro scratch wound healing assay, HUVECs were incubated with $100 \mu \mathrm{M}$ of peptides for $24 \mathrm{~h} .5 \times 10^{4}$ HUVECs per well were seeded in 24-well plates and grown into a monolayer culture with $100 \%$ confluency. After scratching through the monolayer with a pipette tip, wound closure was visually monitored with a Zeiss Axio Scope Inverted Microscope and the ability to recover from wounds (\% closure) was quantified using proprietary software associated with the microscope system.

In vitro angiogenesis/tube formation assays were performed as previously described [18]. Briefly, the impact of peptide hydrogel formulations on in vitro angiogenesis was tested using HUVECs $\left(4 \times 10^{4}\right.$ cells/well $)$ seeded in Matrigel ${ }^{\mathrm{TM}}(50 \mu \mathrm{L})$ coated chambered slides (96-well) containing $100 \mu \mathrm{M}$ of peptide samples, with TCP utilized as control. HUVECs were incubated at $37{ }^{\circ} \mathrm{C}$ for approximately $6 \mathrm{~h}$, and capillary-like structures were imaged using a Zeiss Axio Scope Inverted Microscope.

\subsection{Animal models}

All procedures involving animal experiments were approved by Gazi University Animal Ethics Committee. Suture-induced corneal angiogenesis was used as the neovascularization model, since the cornea is normally an avascular tissue and, therefore, serves as an ideal in vivo model for studying the effects of angiogenesis.

Adult male Sprague-Dawley (SD) rats were exposed to $12 \mathrm{~h}$ light-dark cycles and fed a standard laboratory diet with food and water provided ad libitum. All rats (weighing 200-220 g and aged 8 weeks) were deeply anesthetized prior to surgery. 10-0 nylon sutures were placed at $1-1.5 \mathrm{~mm}$ distance from the temporal limbus of the cornea, and the time of suture placement was considered as day 0 . The suturing operation was performed on only one eye, while the other eye of the animal was left as internal control. Following suture placement, rats were randomized to five groups: Group I $(\mathrm{n}=4)$ treated with Ac-LPPR-Am, Group II $(\mathrm{n}=4)$ treated with $\mathrm{K}_{3}$ PA/E-PA, Group III $(n=4)$ treated with LPPR-PA/E-PA, Group IV $(\mathrm{n}=4)$ treated with bevacizumab and Group $\mathrm{V}(\mathrm{n}=4)$ without any treatment (suture-only group). Corneas were observed for 7 days following the operation to ensure that the suture is successful in stimulating corneal neovascularization and only animals exhibiting neovascularization were subjected to further treatment. LPPR-PA nanofibers and control peptides were delivered to rat corneas by subconjunctival injection (1 cc of $1 \mathrm{mM}$ peptides) on day 7. Similarly, group IV received a subconjunctival injection of $0.1 \mathrm{cc}$ bevacizumab (Avastin ${ }^{\circledR}$ ) at a concentration of $25 \mathrm{mg} / \mathrm{mL}$ on day 7 . Corneal images were taken using a surgical microscope on day 0 (operation day), day 7 (injection day), and day 14. Images were used to measure the corneal surface covered with neovascular vessels. To increase reproducibility, the entire surgery process was performed by a single investigator (B.A.) for every animal. Pupils were dilated with drops of tropicamide $0.5 \%$ (Tropamid $^{\mathbb{R}}$ ) prior to each imaging session [19]. Neovascularization of each cornea was evaluated by an examiner who was blind to the treatment groups. Corneas were then removed and processed for further histological analysis.

\subsection{Histological analysis}

On day 14, all rats were sacrificed and their eyes were fixed with $10 \%$ formalin and embedded in paraffin. Tissue sections $(5 \mu \mathrm{m})$, which were taken with a Leica microtome, were deparaffinized in xylene, rehydrated through graded alcohols to distilled water, and stained with hematoxylin and eosin (H\&E). Endogenous peroxidase was inactivated with $3 \%$ hydrogen peroxide for $10 \mathrm{~min}$ at room temperature, and nonspecific binding sites were blocked with $10 \%$ normal goat serum for $2 \mathrm{~h}$. Antigens were then detected through indirect binding using their respective primary antibodies and HRP-conjugated secondary antibodies. Serial sections were immunolabeled with anti-von Willebrand factor antibody (1:400 dilution; ab6994, Abcam), and a secondary antibody (1:500 dilution; ab6721) to evaluate neovascularization. Sections were developed in 3,3'-diaminobenzidine (DAB) and counterstained with hematoxylin.

The number of blood vessels was counted from at least 6 randomly selected fields in each section. Sections were immunolabeled with anti-von Willebrand factor antibody and quantified by two independent observers under $200 \times$ magnification. Quantifications were performed by the ImageJ analysis system to calculate the number of vessels in each field.

\subsection{Statistical analysis}

Statistical analyses were performed using GraphPad Prism 5. One-way ANOVA was used to compare the differences between the groups and Bonferroni's multiple comparisons test was used for post-hoc correction. Error bars indicate \pm SEM (standard error of mean). At least three independent repeats were performed for each in vitro experiment, with a minimum of $\mathrm{n}=3$ for each repeat. In vivo experiments were performed at a minimum of $\mathrm{n}=4$.

\section{Results}

\subsection{Synthesis and characterization of PAs}

In this study, the LPPR peptide sequence was conjugated to a $\beta$ sheet forming peptide amphiphile molecule to create an effective anti-angiogenic nanofiber system. The well-ordered nanofibrous morphology of this self-assembled peptide system can increase the presentation of the bioactive sequence to the VEGF receptor NRP-1, thereby blocking VEGF's angiogenic effect through competitive exclusion. The soluble LPPR peptide was also synthesized in addition to the amphiphilic form. Lauryl-VVAGKKGLPPR-Am (LPPR-PA) was mixed with lauryl-VVAGE (E-PA) at a 1:1 ratio to form bioactive LPPR-PA nanofibers, while lauryl-VVAGKKK-Am (K $\left.\mathrm{K}_{3}-\mathrm{PA}\right)$ was mixed 1:1 with E-PA to form non-bioactive control nanofibers. Scrambled peptide amphiphile form of LPPR-PA was designed as laurylVVAGKKGPPLR-Am. Since the presence of arginine residue on the surface has previously been shown to be critical for neuropilin-1 affinity, we replaced leucine with proline amino acids in scrambled variant in order to present arginine residues on nanofiber surface. All PA mixtures have a final charge of +1 , resulting from the respective charges of E-PA (at -2), and LPPR-PA, scrambled-PA and $\mathrm{K}_{3}-\mathrm{PA}(\mathrm{at}+3)$ (Fig. S1). Chemical structures of all peptides are given in Fig. 1A. Solid-phase peptide synthesis was used in the production of all peptide molecules used, and the peptide products were characterized by LC-MS and purified with preparative HPLC (Fig. S2).

Circular dichroism (CD) spectroscopy was utilized to analyze the secondary structures associated with formation of nanofibers by peptide amphiphiles and their mixtures [20]. CD results showed that the nanofibrous structures formed by PA molecules contained 
A

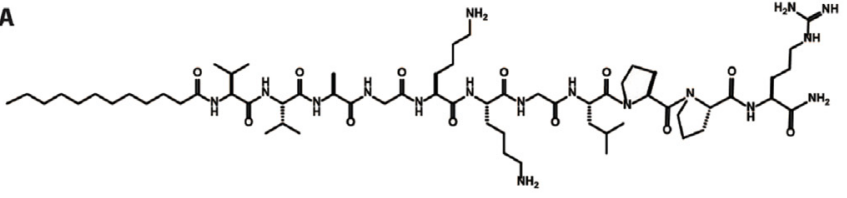

LauryI-WVAGKKGLPPR-Am

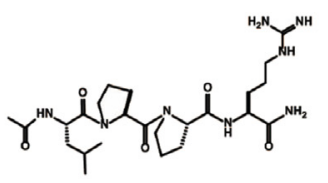

Ac-LPPR-Am

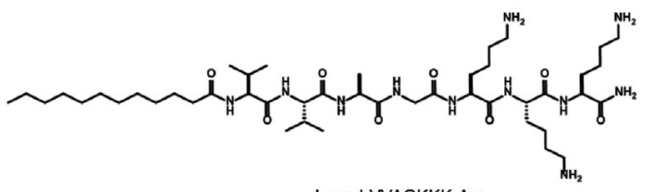

Lauryl-WVAGKKK-Am

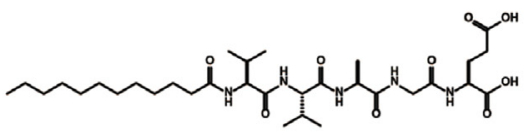

Lauryl-WVAGE

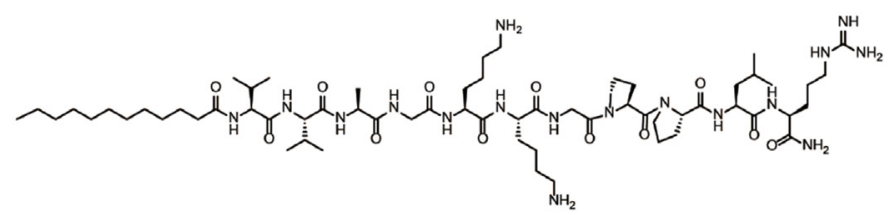

Lauryl-VVAGKKGPPLR-Am

B
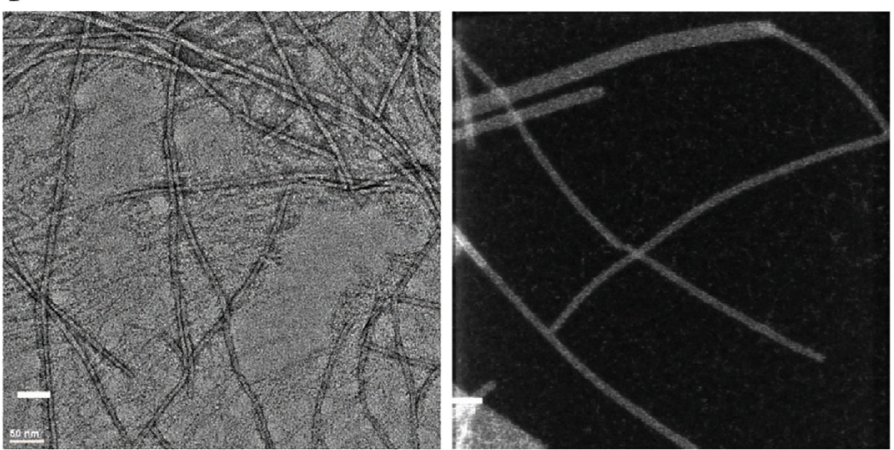

c

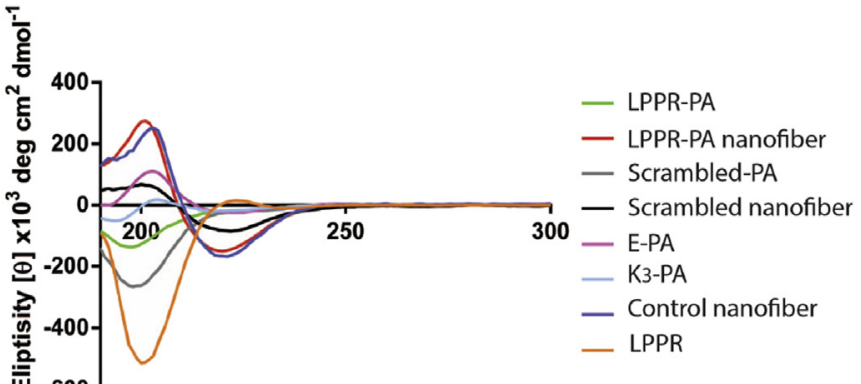

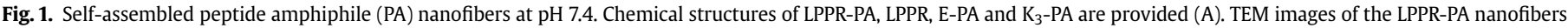

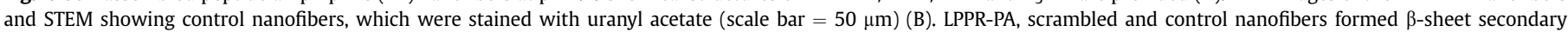
structures analyzed by CD measurements (C). Peptides were dissolved in water and the pH was adjusted with NaOH for TEM, STEM and CD measurements.

$\beta$-sheet structures, as evidenced by their absorbance maxima at around $200 \mathrm{~nm}$ and minima at around $220 \mathrm{~nm}$ (Fig. 1C). TEM and STEM were performed to investigate the morphology of the selfassembled nanostructures in solution and confirmed that the peptide formulations exhibited nanofibrous morphology (Fig. 1B). Both control and LPPR-PA nanofiber structures were $10-20 \mathrm{~nm}$ in diameter and up to several micrometers in length. TEM imaging of the non-amphiphilic LPPR peptide did not yield observable results due to its lack of nanostructure formation. AFM imaging of the scrambled peptide system also revealed a nanofibrous structure, with diameters in the $10-20 \mathrm{~nm}$ range (Fig. S3).

The effect of peptide nanofibers on cellular viability of endothelial cells was analyzed with Live/Dead assay, which demonstrated that LPPR, control, scrambled-PA and LPPR-PA nanofibers did not alter the viability of HUVECs at a concentration of $100 \mu \mathrm{M}$ (Fig. S4A). Cell survival rate of all treatment groups were similar to those on tissue culture plate (TCP) after 24,48 and $72 \mathrm{~h}$ of incubation (Fig. S4B). Even when toxicity assay was performed for higher doses of LPPR-PA nanofibers (up to $1024 \mu \mathrm{M}$ ), no toxicity was observed on HUVECs (Fig. S4C). Overall, these results indicated biocompatibility of control and LPPR-PA nanofibers.

\subsection{LPPR-PA/E-PA treatment reduces endothelial cell proliferation, migration and angiogenic tube formation}

Cell proliferation, migration and tube formation assays were performed to systematically assess the anti-angiogenic effect of
LPPR-PA nanofibers on endothelial cells. Angiogenic regulators, such as VEGF stimulate the proliferation of endothelial cells during blood vessel formation; consequently, the proliferation of HUVECs was evaluated in the presence of VEGF to determine whether the peptide networks were able to disrupt the VEGF/NRP-1 interaction. We demonstrated that LPPR-PA nanofibers inhibited cell proliferation in a dose-dependent manner and with an $\mathrm{IC}_{50}$ of $107 \mu \mathrm{M}$, suggesting that the sequence is able to interfere with endothelial cell growth when incorporated into a peptide nanofiber material (Fig. 2B). Tournaire and colleagues have previously shown that ATWLPPR peptide treatment caused $60 \%$ inhibition in vascular endothelial cell proliferation at a concentration of $420 \mu \mathrm{M}$ [14]. Here, $100 \mu \mathrm{M}$ of LPPR peptide did not cause any effect on the proliferation of HUVECs, while the PA form of this sequence was much more effective in mediating the inhibition of cell proliferation. The inhibitory effect of LPPR-PA was also found to be sequence-specific, since control and scrambled-PA nanofiber did not show any activity at the same concentration (Fig. 2A).

The migration of endothelial cells is an important step of angiogenesis and can be evaluated in vitro by scratch wound healing assay, where a gap is created on a cellular monolayer and its closure is measured to determine the rate of the surrounding cells migrating into the wound [21]. The inhibitory activity of LPPR-PA nanofibers was determined by analyzing the wound closure percentage of HUVECs after $24 \mathrm{~h}$ (Fig. 3). Migration was found to be significantly lower than control in HUVECs treated with LPPR-PA nanofiber in the presence of VEGF. In addition, control nanofibers 


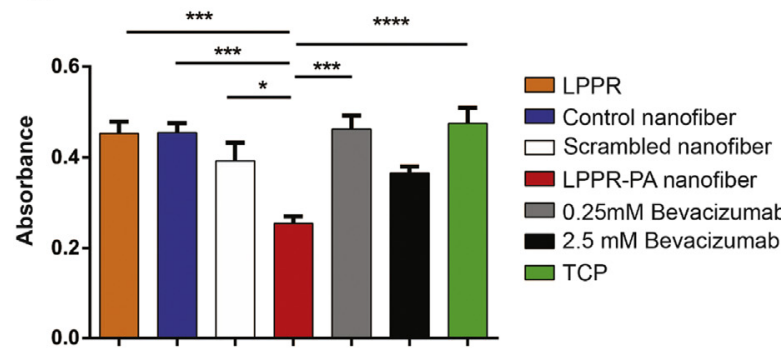

B

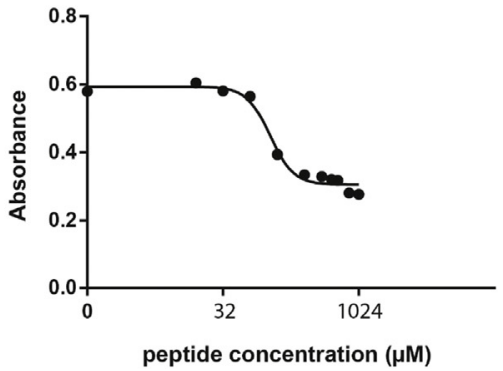

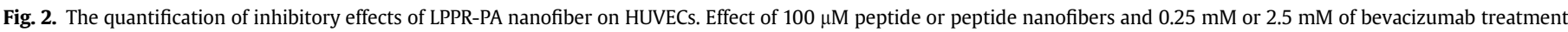

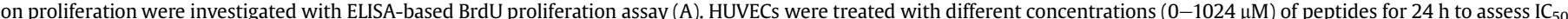

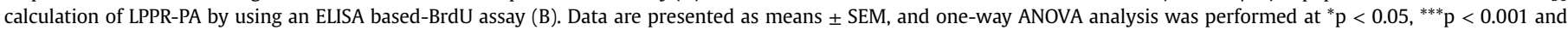
${ }^{* * * *} \mathrm{p}<0.0001$

also inhibited cell migration compared to tissue culture plate (Fig. 3B). Consistent with the proliferation assay results, scratch wound closure measurements demonstrated that VEGF-mediated angiogenesis was markedly blocked by LPPR-PA nanofibers.

During angiogenesis, endothelial cells proliferate and migrate toward an angiogenic growth factor stimulus, and eventually form lumen-bearing tubes to conduct the blood flow. A Matrigel ${ }^{\mathrm{TM}}$ tube formation assay was therefore performed to investigate the antiangiogenic properties of peptide formulations by quantifying their ability to prevent the formation of endothelial tubes. Matrigel $^{\mathrm{TM}}$ contains a mixture of basement membrane components that stimulate endothelial cells to form capillary-like hexagonal structures, and is often preferred over other in vitro assays because of its ease of use and the ability to provide a rapid and quantitative assessment of angiogenesis [22]. Here, growth factor-reduced Matrigel $^{\mathrm{TM}}$ was used with $100 \mu \mathrm{M}$ of VEGF to investigate VEGFinduced tube formation. Angiogenesis assay showed that only non-treated TCP control had well-ordered tube structures (Fig. 4A). Furthermore, tube length measurements were performed to compare the efficacy of treatments in inhibiting angiogenesis. LPPR peptide, control nanofiber, LPPR-PA nanofiber and bevacizumab treatments inhibited tube length by $67.3 \pm 7.19 \%, 73.6 \pm 7.68 \%$, $93.08 \pm 6.19 \%$ and $89.8 \pm 10.1 \%$ compared to TCP, respectively. These data indicated that peptide treatments in general reduced the
A
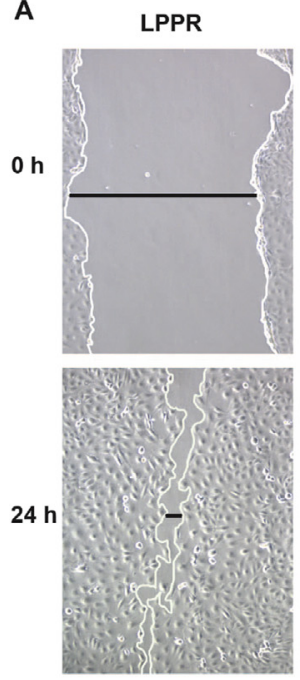

Scrambled nanofiber
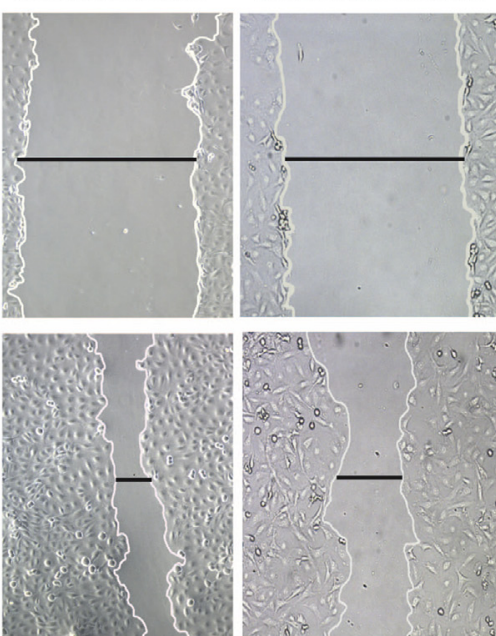

Control nanofiber
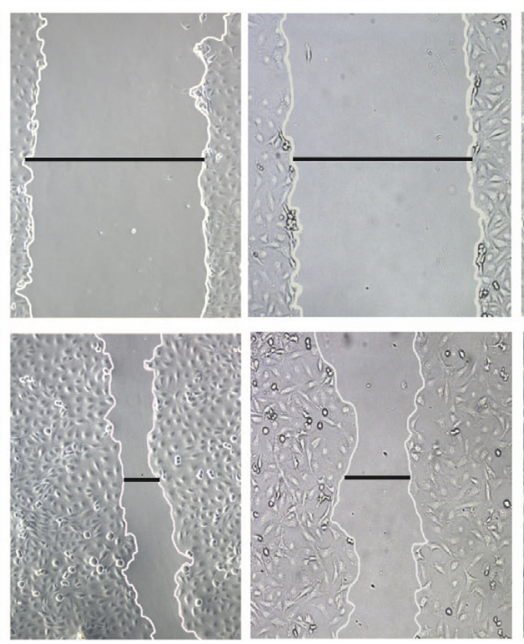

LPPR-PA nanofiber
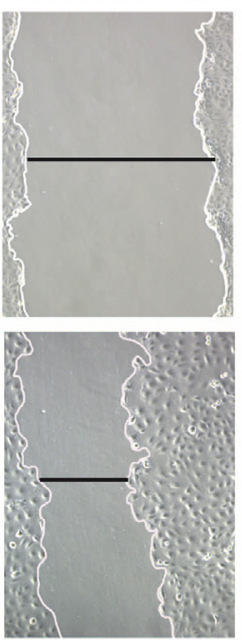

$\varpi$ LPPR

$\square$ Control nanofiber

$\square$ Scrambled nanofiber

$\square$ LPPR-PA nnaofiber

$\square$ TCP
TCP

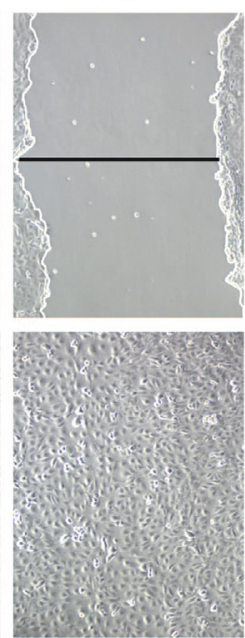

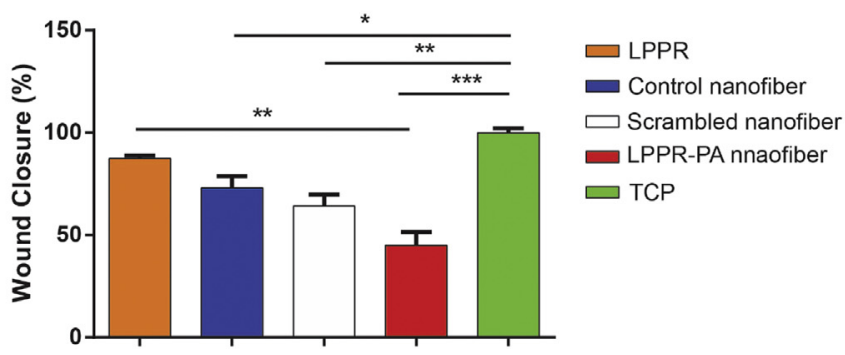

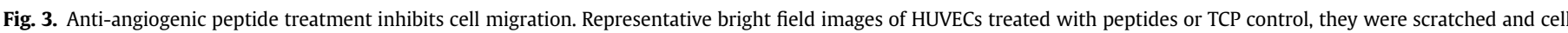

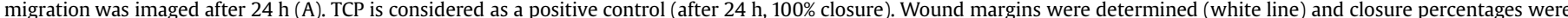

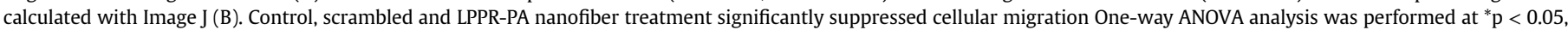
${ }^{* *} \mathrm{p}<0.01$, and ${ }^{* * *} \mathrm{p}<0.001$; images were taken at $100 \times$ magnification. 
tubular assembly of endothelial cells, but LPPR-PA nanofiber treatment caused the most significant inhibition of tube lengths (Fig. 4). The inhibition rate was as strong as bevacizumab treatment, suggesting that LPPR-PA nanofiber treatment was able to suppress VEGF-induced angiogenesis in vitro. In contrast, the scrambled nanofiber did not exhibit any capacity for the inhibition of angiogenesis beyond the LPPR-PA and control nanofibers.

\subsection{The subconjunctival injection of LPPR-PA nanofibers inhibits suture-induced corneal neovascularization}

After demonstrating the anti-angiogenic activity of LPPR-PA nanofibers in vitro, we next investigated the in vivo efficacy of peptide nanofibers using a suture-induced corneal angiogenesis model. As the scrambled PA sequence did not exhibit a substantial anti-angiogenic effect, it was not included in in vivo measurements and a non-bioactive control nanofiber was used instead. Preliminary studies showed that neovascularization began to occur near the corneal suture on day 1 or 2 after surgery and had advanced greatly by day 7 . Consequently, subconjunctival injection of peptides was performed on day 7 to better reflect the clinical presentation of corneal neovascularization, which is typically diagnosed only after advanced neovascularization.

The in vivo anti-angiogenic activity of LPPR-PA nanofibers was evaluated through the measurement of blood vessel growth areas. Corneal neovascularization was induced in all treatment groups with a similar vessel growth area on day 7 (Fig. 5B). Representative images of corneas on day 14 and schematic illustration of in vivo experiments are shown in Fig. S5. The subconjunctival injection of
LPPR-PA nanofiber significantly inhibited corneal neovascularization in rats compared to other treatment group on day 14 (Fig. 5). Moreover, the efficacy of the peptide nanofiber was higher than the LPPR peptide and bevacizumab: Bevacizumab showed a $51.2 \%$ inhibition of corneal neovascularization in our rat model, while LPPR-PA nanofiber treatment resulted in around $81.3 \%$ inhibition relative to untreated controls. The efficacy of subconjunctival bevacizumab treatment in this model was similar to its efficacy in humans, suggesting that LPPR-PA nanofiber may be a better alternative for corneal neovascularizaiton therapy [23]. However, further studies are required to compare the safety of nanofiber injections and conclusively demonstrate the advantage of anti-angiogenic peptides for the treatment of corneal neovascularization.

The effects of the anti-angiogenic peptide nanofibers on new vessel formation were further characterized through von Willebrand factor staining on day 14 after surgery. The total number of vessels was calculated from both central and peripheral areas of the corneal surface. Histological and quantitative analyses of the cornea revealed that LPPR-PA nanofiber treatment led to a significant reduction in the number of blood vessels compared to untreated control (Fig. 6). Although corneal neovascularization was not completely regressed, only a small number of vessels had remained in the peripheral area after a single injection of LPPR-PA. These data, in tandem with vessel growth area calculations, indicate that VEGF suppression plays an important role in the inhibition of corneal angiogenesis, and that LPPR-PA treatment can effectively reduce corneal neovascularization through this process.

A
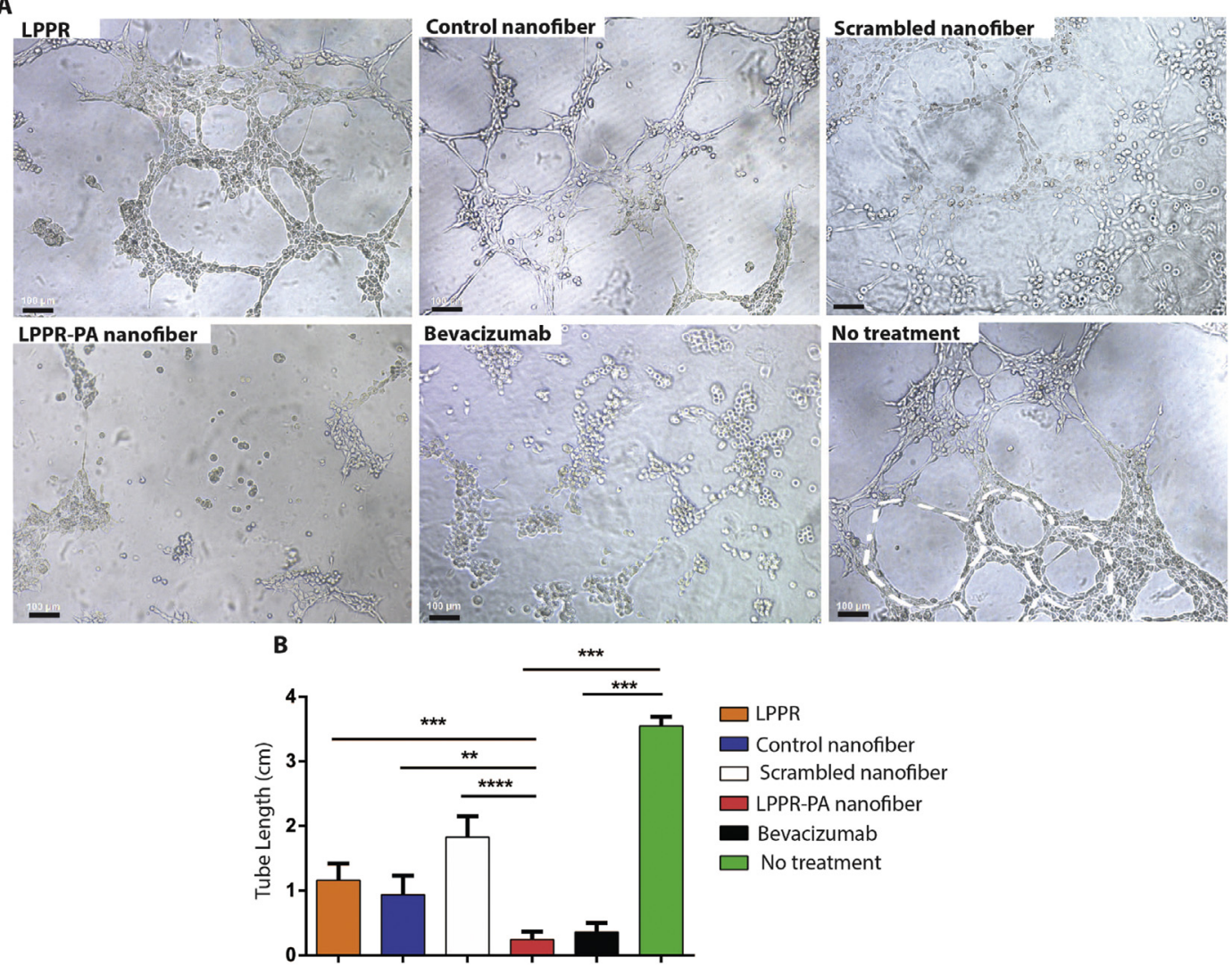

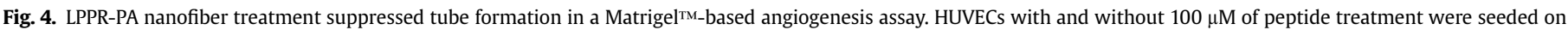

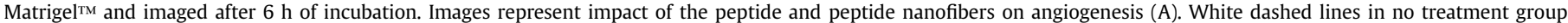

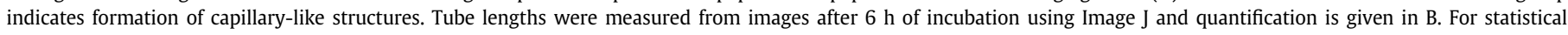
analysis, one-way ANOVA was performed at ${ }^{* *} \mathrm{p}<0.01,{ }^{* * *} \mathrm{p}<0.001$ and ${ }^{* * * *} \mathrm{p}<0.0001$. Scale bars are $100 \mu \mathrm{m}$. 

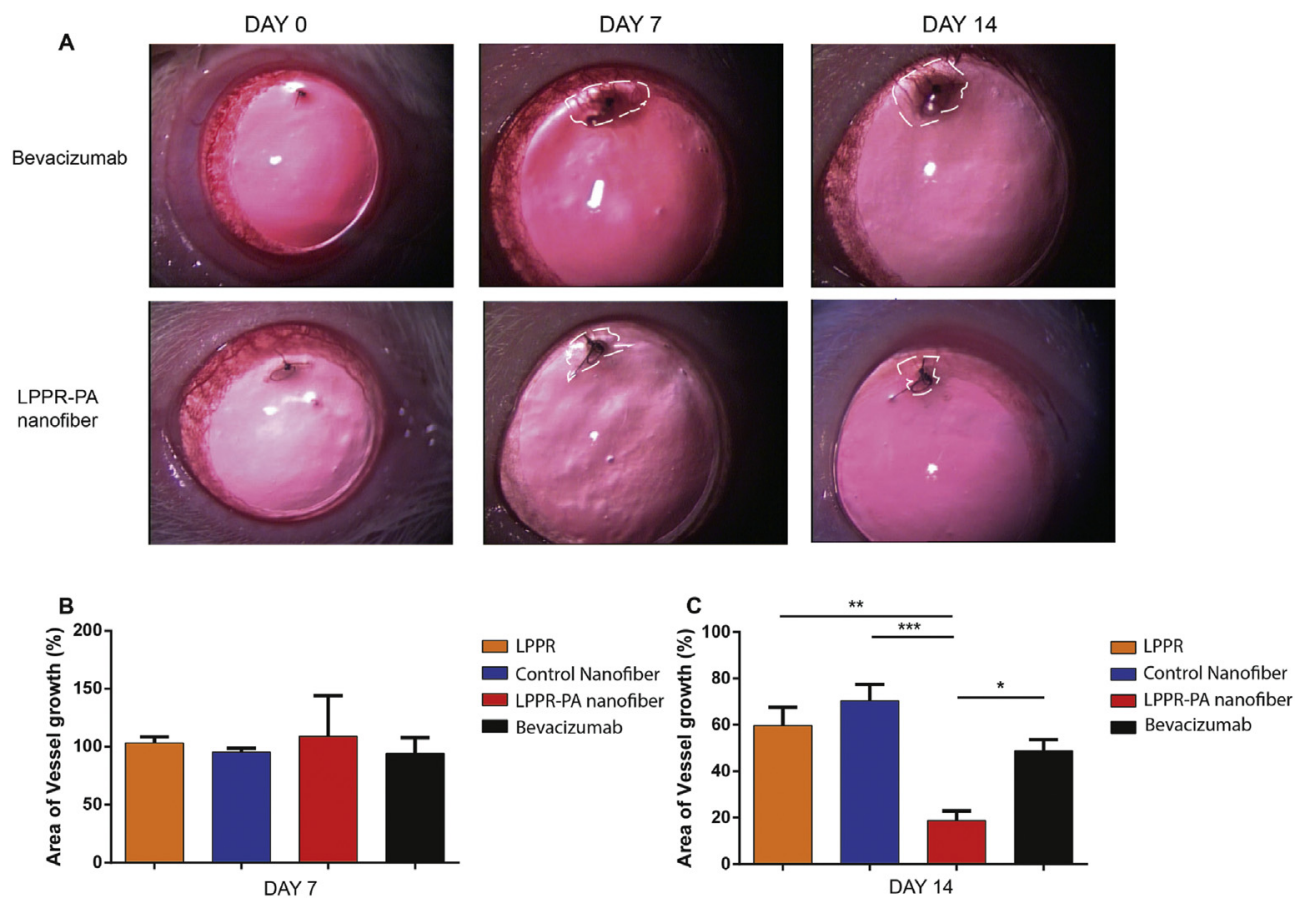

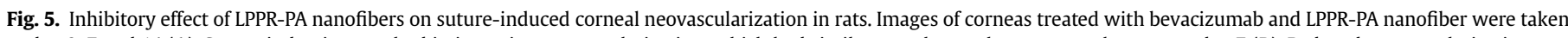

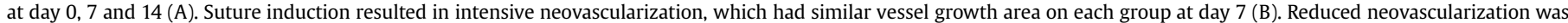

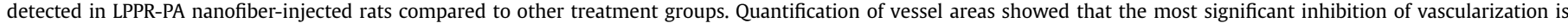
found in the anti-angiogenic nanofiber treated group (B). One-way ANOVA analysis was performed at ${ }^{*} \mathrm{p}<0.05,{ }^{* *} \mathrm{p}<0.01$, and ${ }^{* * *} \mathrm{p}<0.001$.

\section{Discussion}

Disruption of aberrant VEGF signaling is the most common strategy for the treatment of corneal neovascularization, which aims to facilitate the regression and elimination of abnormal blood vessels. Since the angiogenic activity of VEGF is mediated by its receptors, the blocking of receptor-ligand interactions is a promising approach for anti-VEGF therapies. In this study, the LPPR sequence was incorporated into a peptide amphiphile sequence capable of self-assembling into a transparent and stable nanofiber matrix [24]. The LPPR-PA molecule contains hydrophilic head and hydrophobic tail regions, which mediate self-assembly through both electrostatic interactions and hydrophobic collapse to form ordered structures. The mixing of LPPR-PA with oppositely charged E-PA formed stable nanofibers. TEM and CD analyses of peptide amphiphiles confirmed that the PA molecules formed $\beta$-sheet-rich nanofiber structures (Fig. 1). The bioavailability and biocompatibility of peptide assemblies are critical for designing nanotherapeutic systems, and factors such as concentration, hydrogen bonding and charge may severely affect the biocompatibility of peptides $[25,26]$. Consequently, the biocompatibility of the PA nanofibers was investigated using Live-Dead assay and both LPPRPA and controls were found not to be toxic to cells at a concentration of $100 \mu \mathrm{M}$ (Fig. 2).

Anti-angiogenic properties of LPPR-PA nanofibers were investigated in vitro by cell proliferation, migration and tube formation assays. Effects of LPPR-PA nanofibers on angiogenesis were compared to LPPR peptide, control and scrambled PA nanofibers. LPPR-PA nanofiber had significant inhibitory effect on cell proliferation in a dose-dependent manner. In contrast, the soluble LPPR peptide inhibited cellular growth only to a lesser extent, possibly because the nanofibrous architecture of peptide amphiphile systems improves the efficiency of the bioactive sequence by mediating its presentation to NRP-1. In addition, resistance of nanofiber systems to proteolytic degradation may contribute to their efficiency [27,28]. The binding of VEGF to NRP-1 was reported to be essential for VEGF-induced cell migration; consequently, an in vitro scratch wound healing assay was performed to examine the role of the LPPR sequence on cell migration [29,30]. LPPR-PA nanofiber treatment was found to significantly inhibit wound closure by HUVECs in $24 \mathrm{~h}$, suggesting that the bioactive nanofibers are able to effectively prevent VEGF-induced cellular migration (Fig. 3). Although migration was markedly inhibited by the LPPR-PA nanofiber, the control nanofiber also surprisingly exhibited a suppressive effect compared to TCP (Fig. 4). This effect may have been caused by the multivalent presentation of charged residues on peptide nanofibers, which can limit the migration of endothelial cells and allow control nanofibers to display some measure of antiangiogenic effect. However, peptide sequence is essential for antiangiogenic activity since LPPR-PA nanofiber was able to inhibit migration more efficiently than the scrambled-PA nanofiber.

HUVECs form capillary-like tubes when stimulated with angiogenic factors, and this property can be used to assess the antiangiogenic properties of a broad variety of materials [31]. The angiogenesis assay demonstrated that peptide nanofiber treatment significantly reduced tube formation in the presence of VEGF, exhibiting a suppressive effect comparable to the commercially available drug bevacizumab (Fig. 4). Control nanofibers also exhibited an inhibitory effect on tube formation, which normally requires the clustering of endothelial cells and may have been blocked by the prevention of cellular migration induced by control peptides. This result reveals that charge and structural properties also had effects on bioactivity of the material. However, comparison of activity of LPPR-PA on angiogenesis with control and scrambledPA showed that the sequence itself plays an important role.

While peptide sequences (including the LPPR motif) have previously been reported to inhibit endothelial cell proliferation and tube formation; peptide nanofibers described in the present study were observed to inhibit corneal angiogenesis to a greater extent than the soluble peptides, potentially because of the ability of well- 


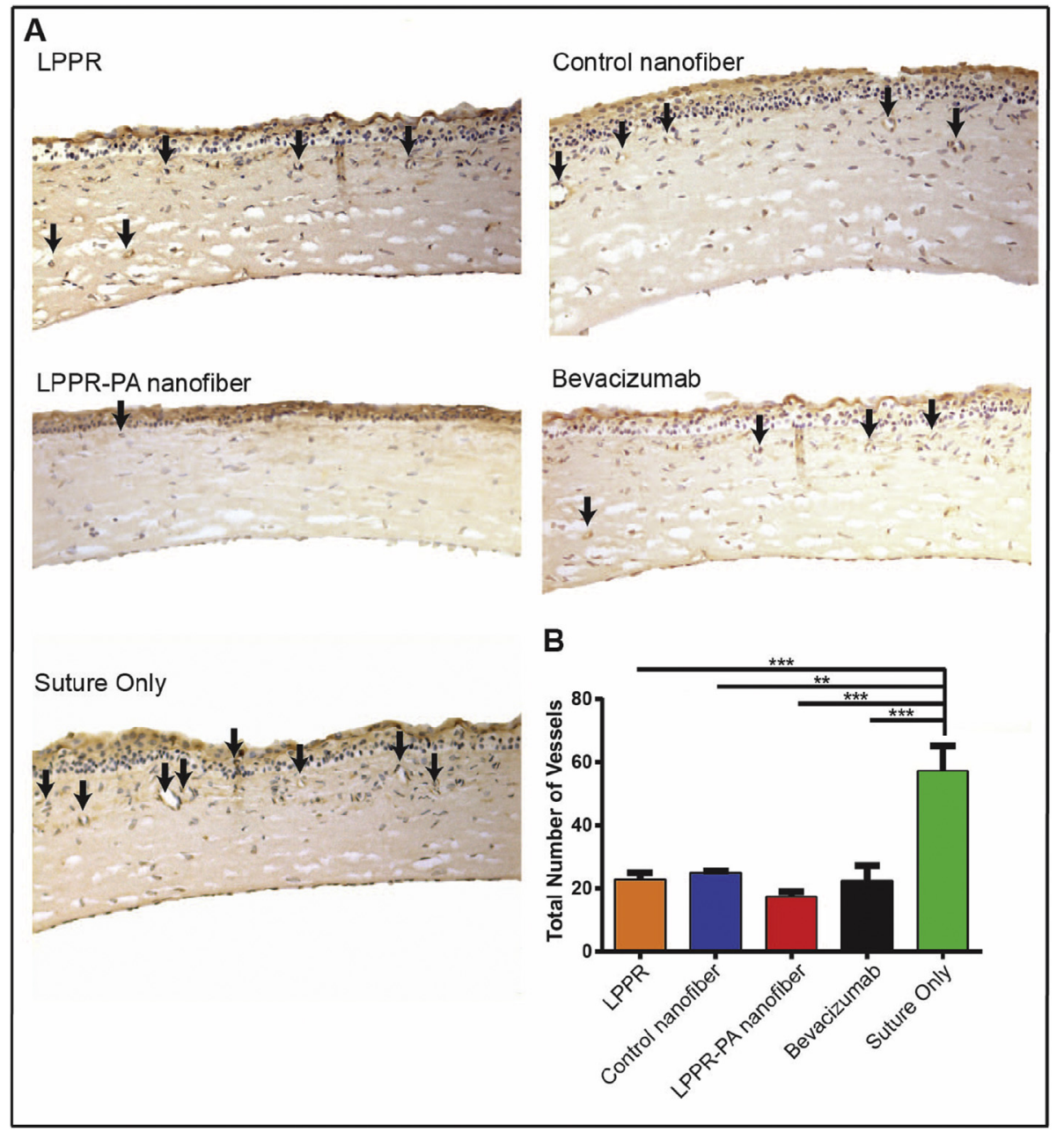

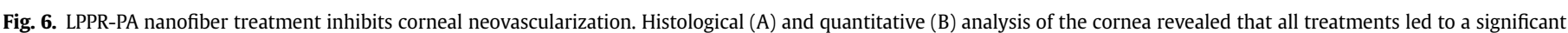

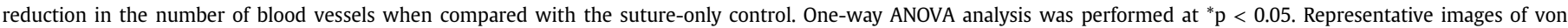

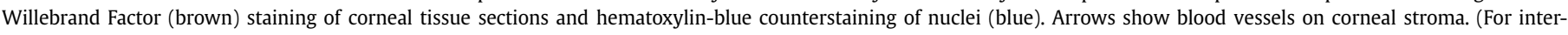
pretation of the references to colour in this figure legend, the reader is referred to the web version of this article.)

organized peptide nanofibers to present their bioactive sequences in a coordinated and multivalent manner [14,17]. In addition, peptide network treatment resulted in a substantially greater reduction in blood vessel numbers compared to the commercially available drug bevacizumab, suggesting that the LPPR-PA nanofiber system may be utilized as a novel therapeutic agent against corneal neovascularization.

The efficacy of nanofiber peptide treatment in cornea was investigated in vivo by using suture-induced angiogenesis model. This model is well-established in rats, sees widespread use in neovascularization research and has been shown to provide a quantitative and reproducible assessment of in vivo angiogenesis [32-34]. In vivo studies showed that LPPR-PA reduced corneal neovascularization more effectively than LPPR peptide and bevacizumab (Fig. 5). This result was supported through the quantification of the number of blood vessels by von Willebrand staining of corneal tissue sections. While we have demonstrated that LPPR-PA nanofibers inhibit angiogenesis in vitro and reduce corneal vascularization in vivo, further studies are necessary to confirm the effectiveness of the material as a therapeutic agent.

It is well-known that the maintenance of the avascular and transparent structure of the cornea depends strongly on the balance between angiogenic and anti-angiogenic factors, and that VEGF plays a crucial role in this process. Due to its strong and highly specific affinity to VEGF, LPPR-PA might be utilized for the treatment of excessive vascularization associated with this growth factor. However, in disorders involving multiple growth factors, treatment with other anti-angiogenic molecules, such as anti-IGF-1 (Insulin-like growth factor-1), anti-PDGF (platelet derived growth factor), PEDF (pigment epithelium-derived factor) and endostatin, may be combined with LPPR-PA application for better prognosis.

\section{Conclusion}

Corneal neovascularization is associated with severe visual impairment and its rapid and effective treatment is of great importance for restoring the sight of the affected. The peptide nanofiber system described in the present study is able to reduce corneal blood vessel areas even after extensive vascularization had already taken place, and was as effective as a commercial agent in this capacity. Comparison of LPPR-PA nanofiber and LPPR peptide treatments suggested that the nanofiber form is more effective in reducing angiogenesis than the soluble peptide, possibly because of the ordered organization of the bioactive sequences in peptide nanofibers. A sustained release effect may also be responsible for this phenomenon, as the soluble peptide is more susceptible to proteolytic action and may be removed from the cornea with time. Overall, data from a variety of in vitro and in vivo experimental 
approaches suggest that LPPR-PA nanofibers effectively inhibit endothelial cell proliferation and migration, aberrant capillary formation and neovascularization in the eye, and may be utilized as a novel treatment against corneal neovascularization and possibly other angiogenesis-related disorders.

\section{Acknowledgements}

B.S. is supported by TUBITAK BIDEB PhD fellowship. The authors thank Alper Devrim Ozkan for AFM imaging and his valuable comments on the manuscript and Zeynep Erdogan for her technical help in LC-MS studies.

\section{Appendix A. Supplementary data}

Supplementary data related to this article can be found at http:// dx.doi.org/10.1016/j.biomaterials.2016.08.045.

\section{References}

[1] L.S. Gambino, N.G. Wreford, J.F. Bertram, P. Dockery, F. Lederman, P.A. Rogers, Angiogenesis occurs by vessel elongation in proliferative phase human endometrium, Hum. Reprod. 17 (5) (2002) 1199-1206.

[2] M.S. Pepper, Manipulating angiogenesis. From basic science to the bedside, Arterioscler. Thromb. Vasc. Biol. 17 (4) (1997) 605-619.

[3] S. Maddula, D.K. Davis, M.K. Burrow, B.K. Ambati, Horizons in therapy for corneal angiogenesis, Ophthalmology 118 (3) (2011) 591-599.

[4] D.R. Mould, K.R. Sweeney, The pharmacokinetics and pharmacodynamics of monoclonal antibodies-mechanistic modeling applied to drug development, Curr. Opin. Drug Discov. Devel 10 (1) (2007) 84-96.

[5] H.S. Chu, T.C. Chen, F.R. Hu, W.L. Chen, Recurrence of corneal neovascularization associated with lipid deposition after subconjunctival injection of bevacizumab, Cornea 32 (11) (2013) 1446-1453.

[6] P. Chames, M. Van Regenmortel, E. Weiss, D. Baty, Therapeutic antibodies: successes, limitations and hopes for the future, Br. J. Pharmacol. 157 (2) (2009) 220-233.

[7] H. Cui, M.J. Webber, S.I. Stupp, Self-assembly of peptide amphiphiles: from molecules to nanostructures to biomaterials, Biopolymers 94 (1) (2010) 1-18.

[8] E. Busseron, Y. Ruff, E. Moulin, N. Giuseppone, Supramolecular self-assemblies as functional nanomaterials, Nanoscale 5 (16) (2013) 7098-7140.

[9] E. Arslan, I.C. Garip, G. Gulseren, A.B. Tekinay, M.O. Guler, Bioactive supramolecular peptide nanofibers for regenerative medicine, Adv. Healthc. Mater 3 (9) (2014) 1357-1376.

[10] D. Mumcuoglu, M. Sardan, T. Tekinay, M.O. Guler, A.B. Tekinay, Oligonucleotide delivery with cell surface binding and cell penetrating Peptide amphiphile nanospheres, Mol. Pharm. 12 (5) (2015) 1584-1591.

[11] R.H. Zha, S. Sur, J. Boekhoven, H.Y. Shi, M. Zhang, S.I. Stupp, Supramolecular assembly of multifunctional maspin-mimetic nanostructures as a potent peptide-based angiogenesis inhibitor, Acta Biomater. 12 (2015) 1-10.

[12] K.N. Sulochana, R. Ge, Developing antiangiogenic peptide drugs for angiogenesis-related diseases, Curr. Pharm. Des. 13 (20) (2007) 2074-2086.

[13] C. Rüegg, M. Hasmim, F.J. Lejeune, G.C. Alghisi, Antiangiogenic peptides and proteins: from experimental tools to clinical drugs, Biochim. Biophys. Acta 1765 (2) (2006) 155-177.

[14] R. Binétruy-Tournaire, C. Demangel, B. Malavaud, R. Vassy, S. Rouyre M. Kraemer, J. Plouët, C. Derbin, G. Perret, J.C. Mazié, Identification of a peptide blocking vascular endothelial growth factor (VEGF)-mediated angiogenesis, EMBO J. 19 (7) (2000) 1525-1533.
[15] A. Starzec, P. Ladam, R. Vassy, S. Badache, N. Bouchemal, A. Navaza, C.H. du Penhoat, G.Y. Perret, Structure-function analysis of the antiangiogenic ATWLPPR peptide inhibiting VEGF(165) binding to neuropilin-1 and molecular dynamics simulations of the ATWLPPR/neuropilin-1 complex, Peptides 28 (12) (2007) 2397-2402.

[16] R.J. Giordano, M. Cardó-Vila, A. Salameh, C.D. Anobom, B.D. Zeitlin, D.H. Hawke, A.P. Valente, F.C. Almeida, J.E. Nör, R.L. Sidman, R. Pasqualini, W. Arap, From combinatorial peptide selection to drug prototype (I): targeting the vascular endothelial growth factor receptor pathway, Proc. Natl. Acad. Sci. U. S. A. 107 (11) (2010) 5112-5117.

[17] A. Starzec, R. Vassy, A. Martin, M. Lecouvey, M. Di Benedetto, M. Crépin, G.Y. Perret, Antiangiogenic and antitumor activities of peptide inhibiting the vascular endothelial growth factor binding to neuropilin-1, Life Sci. 79 (25) (2006) 2370-2381.

[18] R. Mammadov, B. Mammadov, S. Toksoz, B. Aydin, R. Yagci, A.B. Tekinay M.O. Guler, Heparin mimetic peptide nanofibers promote angiogenesis, Biomacromolecules 12 (10) (2011) 3508-3519.

[19] Y. Geng, K.P. Greenberg, R. Wolfe, D.C. Gray, J.J. Hunter, A. Dubra, J.G. Flannery, D.R. Williams, J. Porter, In vivo imaging of microscopic structures in the rat retina, Investig. Ophthalmol. Vis. Sci. 50 (12) (2009) 5872-5879.

[20] N.J. Greenfield, Using circular dichroism spectra to estimate protein secondary structure, Nat. Protoc. 1 (6) (2006) 2876-2890.

[21] C.C. Liang, A.Y. Park, J.L. Guan, In vitro scratch assay: a convenient and inexpensive method for analysis of cell migration in vitro, Nat. Protoc. 2 (2) (2007) 329-333.

[22] C.P. Khoo, K. Micklem, S.M. Watt, A comparison of methods for quantifying angiogenesis in the Matrigel assay in vitro, Tissue Eng. Part C Methods 17 (9) (2011) 895-906.

[23] A.A. Zaki, S.F. Farid, Subconjunctival bevacizumab for corneal neovascularization, Acta Ophthalmol. 88 (8) (2010) 868-871.

[24] A. Dehsorkhi, V. Castelletto, I.W. Hamley, Self-assembling amphiphilic peptides, J. Pept. Sci. 20 (7) (2014) 453-467.

[25] C.J. Newcomb, S. Sur, J.H. Ortony, O.S. Lee, J.B. Matson, J. Boekhoven, J.M. Yu, G.C. Schatz, S.I. Stupp, Cell death versus cell survival instructed by supramolecular cohesion of nanostructures, Nat. Commun. 5 (2014) 3321.

[26] K. Saar, M. Lindgren, M. Hansen, E. Eiríksdóttir, Y. Jiang, K. Rosenthal-Aizman, M. Sassian, U. Langel, Cell-penetrating peptides: a comparative membrane toxicity study, Anal. Biochem. 345 (1) (2005) 55-65.

[27] Y.F. Tian, G.A. Hudalla, H. Han, J.H. Collier, Controllably degradable $\beta$-sheet nanofibers and gels from self-assembling depsipeptides, Biomater. Sci. 1 (10) (2013).

[28] S. Zhang, T. Holmes, C. Lockshin, A. Rich, Spontaneous assembly of a selfcomplementary oligopeptide to form a stable macroscopic membrane, Proc. Natl. Acad. Sci. U. S. A. 90 (8) (1993) 3334-3338.

[29] B. Herzog, C. Pellet-Many, G. Britton, B. Hartzoulakis, I.C. Zachary, VEGF binding to NRP1 is essential for VEGF stimulation of endothelial cell migration, complex formation between NRP1 and VEGFR2, and signaling via FAK Tyr407 phosphorylation, Mol. Biol. Cell 22 (15) (2011) 2766-2776.

[30] Q. Pan, Y. Chanthery, W.C. Liang, S. Stawicki, J. Mak, N. Rathore, R.K. Tong, J. Kowalski, S.F. Yee, G. Pacheco, S. Ross, Z. Cheng, J. Le Couter, G. Plowman, F. Peale, A.W. Koch, Y. Wu, A. Bagri, M. Tessier-Lavigne, R.J. Watts, Blocking neuropilin-1 function has an additive effect with anti-VEGF to inhibit tumor growth, Cancer Cell 11 (1) (2007) 53-67.

[31] A.M. Goodwin, In vitro assays of angiogenesis for assessment of angiogenic and anti-angiogenic agents, Microvasc. Res. 74 (2-3) (2007) 172-183.

[32] J.N. Kather, J. Kroll, Transgenic mouse models of corneal neovascularization: new perspectives for angiogenesis research, Investig. Ophthalmol. Vis. Sci. 55 (11) (2014) 7637-7651.

[33] F. Wang, Y.P. Deng, M. Zhang, Effect of KH902 in inhibiting suture induced corneal neovascularization, Sichuan Da Xue Xue Bao Yi Xue Ban. 45 (3) (2014) 419-423.

[34] Z. Li, L. Yao, J. Li, W. Zhang, X. Wu, Y. Liu, M. Lin, W. Su, Y. Li, D. Liang, Celastrol nanoparticles inhibit corneal neovascularization induced by suturing in rats, Int. J. Nanomedicine 7 (2012) 1163-1173. 